\title{
MicroRNA-452 suppresses pancreatic cancer migration and invasion by directly targeting B-cell-specific Moloney murine leukemia virus insertion site 1
}

\author{
HONGYAN LI, YAN WU and PEIXIU LI \\ Department of Endocrinology, Weifang People's Hospital, Weifang, Shandong 261041, P.R. China \\ Received November 23, 2015; Accepted April 28, 2017
}

DOI: $10.3892 / \mathrm{ol} .2017 .6566$

\begin{abstract}
Pancreatic cancer, one of the most common cancers globally, is the fourth most common cause of cancer-associated mortality in the USA. The 5-year relative survival rate for patients with pancreatic cancer is $\sim 5 \%$ and the median survival time is only 6 months. The poor prognosis is mainly due to early and aggressive local invasion and metastasis, as well as dissemination of the pancreatic cancer cells. The present study demonstrated that microRNA-452 (miR-452) was markedly downregulated in pancreatic cancer tissues, particularly in metastatic tumors and pancreatic cancer cell lines. Overexpression of miR-452 significantly inhibited migration and invasion in pancreatic cancer cells. In addition, the molecular mechanism underlying the inhibitory functions of miR-452 in pancreatic cancer was also investigated. The results indicated that B-cell-specific Moloney murine leukemia virus insertion site 1 (BMI1) was a direct target gene of miR-452 in pancreatic cancer. Overexpression of miR-452 inhibited the migration and invasion of pancreatic cancer, at least partially by knockdown of BMI1 expression. The results provided novel insight with potential therapeutic applications for the treatment of metastatic pancreatic cancer.
\end{abstract}

\section{Introduction}

Pancreatic cancer, one of the most common cancers globally, is the fourth most common cause of cancer-associated mortality in the USA (1). In 2015, it was estimated that there would be 48,960 new pancreatic cancer cases and 40,560 mortalities due to pancreatic cancer in the USA (2). Epidemiological studies have demonstrated that the likelihood of pancreatic cancer is associated with numerous factors, including smoking, high-fat

Correspondence to: Professor Peixiu Li, Department of Endocrinology, Weifang People's Hospital, 151 Guangwen Road, Kuiwen, Weifang, Shandong 261041, P.R. China

E-mail: lipeixiu041@163.com

Key words: pancreatic cancer, pancreatic ductal adenocarcinoma, microRNA-452, B-cell-specific Moloney murine leukemia virus insertion site 1 , metastasis, therapy high-protein diets, alcoholism, coffee drinking, exposure to certain chemical carcinogens, diabetes mellitus and chronic pancreatitis $(3,4)$. Pancreatic ductal adenocarcinoma is the most common subtype of pancreatic cancer, accounting for $85-90 \%$ of all pancreatic cancer cases (5). Although progress has been made in surgery and perioperative management, the 5-year relative survival rate for patients with pancreatic cancer remains at $\sim 5 \%$ and the median survival time is only 6 months (6). The poor prognosis is mainly a result of early and aggressive local invasion and metastasis, as well as dissemination of the pancreatic cancer cells (7). Therefore, it is urgent to improve understanding of the molecular mechanisms underlying the metastasis of pancreatic cancer, and to investigate more effective therapeutic treatments for patients with pancreatic cancer to block cancer metastasis.

MicroRNAs (miRNAs/miRs) are considered to be useful biomarkers for the early diagnosis, therapy and prognosis of human cancers, including pancreatic cancer (8-10). They are an evolutionarily conserved group of non-protein-coding and short RNAs, 18-22 nucleotides in length, whose function is to regulate the expression of their target protein-coding genes either by translational suppression or gene degradation through binding to the 3 ' untranslated regions (3'UTRs) of target genes in a base-pairing manner $(11,12)$. An increasing number of studies have demonstrated that miRNAs regulate numerous biological processes, including cell growth, apoptosis, the cell cycle, metastasis and metabolism, and consequently, their alterations are considered to perform important functions in carcinogenesis and progression of cancers (13). Abnormal expression of miRNAs is associated with various types of diseases, including cancer, and tumor-associated miRNAs act as tumor suppressors or oncogenes depending on their target mRNAs (14). Therefore, elucidating the expression, function and molecular mechanism of miRNAs will be particularly useful in investigating novel therapeutic treatments for cancers.

miR-452 has been studied in several types of cancer, including non-small cell lung cancer (15), breast cancer (16), bladder cancer (17) and hepatocellular carcinoma (18). However, the expression, biological function and molecular mechanism of miR-452 in pancreatic cancer remains to be fully elucidated. The present study revealed that miR-452 was significantly downregulated in pancreatic cancer tissues, particularly in metastatic tumors and pancreatic cancer cell lines. Notably, migration and invasion assays indicated that 
overexpression of miR-452 decreased the migration and invasion of pancreatic cancer cells. Subsequent experiments demonstrated that BMI1 was a direct target gene of miR-452 in pancreatic cancer. Collectively, miR-452 suppressed the migration and invasion of pancreatic cancer cells by directly targeting BMI1.

\section{Materials and methods}

Human tissue specimens and ethics statement. A total of 32 pancreatic cancer tissues with matched adjacent normal tissues were obtained from patients (male, 19; female, 13; age, 46-69 years; mean, 57 years) during surgery at Weifang People's Hospital (Weifang, China) between June 2013 and March 2015. None of patients underwent chemotherapy or radiotherapy prior to surgery. All the tissues were immediately snap-frozen and stored in liquid nitrogen until use. For the analyzed tissue specimens, written informed consent was obtained from all patients to use excess pathological specimens for study purposes. The present study was approved by the Ethics Committee of Weifang People's Hospital and performed according to the ethical principles.

Cell culture and cell transfection. The human pancreatic cancer cell lines (PANC-1, SW1990, ASPC-1 and CFPAC-1) and the human normal pancreatic cell line (HPDE6c7) were obtained from American Type Culture Collection (Manassas, VA, USA) and maintained in Dulbecco's modified Eagle's medium supplemented with $10 \%$ fetal bovine serum (FBS), $2 \mathrm{mM}$ glutamine, $100 \mathrm{IU} / \mathrm{ml}$ penicillin and $100 \mu \mathrm{g} / \mathrm{ml}$ streptomycin (all from Gibco; Thermo Fisher Scientific, Inc., Waltham, MA, USA). All cell lines were cultured in a humidified cell incubator, with an atmosphere of $5 \% \mathrm{CO}_{2}$ and $95 \%$ air, at $37^{\circ} \mathrm{C}$.

miR-452 mimic and negative control (NC) were obtained from Shanghai GenePharma Co., Ltd. (Shanghai, China). BMI1 small interfering RNA (siRNA) and NC siRNA were purchased from Guangzhou RiboBio Co., Ltd. (Guangzhou, China). The miR-452 mimic sequence was 5'-AACUGU UUGCAGAGGAAACUGA-3' and the NC sequence was 5'-UUCUCCGAACGUGUCACGUTT-3'. The BMI1 siRNA sequence was 5'-GUUCACAAGACCAGACCAC-3' and the NC siRNA sequence was 5'-UUCUCCGAACGUGUCACG UTT-3'. miRNA or siRNA were transfected into cells using Lipofectamine 2000 (Invitrogen; Thermo Fisher Scientific, Inc.) in accordance with the manufacturer's protocol. The medium was replaced with fresh culture medium 6-8 $\mathrm{h}$ after transfection.

Reverse transcription-quantitative polymerase chain reaction $(R T-q P C R)$. Total RNA was isolated from tissues and cell lines using TRIzol regent (Invitrogen; Thermo Fisher Scientific, Inc.), according to the manufacturer's protocol. For miRNA expression, RT-qPCR assays were performed using a TaqMan miRNA Assay in an Applied Biosystems 7500 Real-time PCR system (both from Applied Biosystems; Thermo Fisher Scientific, Inc.). For mRNA expression, cDNA was generated from total RNA through reverse transcription using M-MLV reverse transcriptase (Promega Corporation, Madison, WI, USA). BMI1 mRNA expression was evaluated with
SYBR-Green Master Mix (Takara Biotechnology Co., Ltd., Dalian, China). The thermocycling conditions were as follows: $95^{\circ} \mathrm{C}$ for $10 \mathrm{~min}$, followed by 40 cycles of $95^{\circ} \mathrm{C}$ for $15 \mathrm{sec}$ and $60^{\circ} \mathrm{C}$ for $1 \mathrm{~min}$. The primers were designed as follows: miR-452, 5'-GCGAACTGTTTGCAGAGG-3' (forward) and 5'-CAGTGCGTGTCGTGGAGT-3' (reverse); U6, 5'-CTCGCT TCGGCAGCACA-3' (forward) and 5'-ACGCTTCACCGA ATTTGAGT-3' (reverse); BMI1, 5'-GTGCTTTGTGGAGGG TACTTCAT-3' (forward) and 5'-TTGGACATCACAAAT AGGACAATACTT-3' (reverse); and GAPDH, 5'-GAAGGT GAAGGTCGGAGTC-3' (forward) and 5'-GAAGATGGT GATGGGATTTC-3' (reverse). Each sample was analyzed in triplicate. U6 and GADPH were used as internal controls for miR-452 and BMI1 mRNA expression, respectively. Relative expression levels were calculated using the $2^{-\Delta \Delta C \mathrm{a}}$ method (19).

Migration and invasion assay. Transwell chambers (Costar; Corning Incorporated, Corning, NY, USA) with 8-mm pore size polycarbonate membranes were applied to examine cell migration and invasion capacity. For the invasion assay, Transwell chambers were coated with Matrigel $(40 \mu \mathrm{g} / \mathrm{well}$; BD Biosciences, San Jose, CA, USA) prior to the experiment. In brief, $1 \times 10^{5}$ transfected cells suspended in $200 \mu 1$ serum-free medium were placed in the top chambers and $500 \mu \mathrm{l}$ complete culture medium containing 10\% FBS was added to the lower chambers.

Following incubation at $37^{\circ} \mathrm{C}$ in a cell incubator for $48 \mathrm{~h}$, the cells on the top surface of the Transwell chamber were gently removed from the top chambers with a cotton swab. Cells on the lower surface of the membrane were then fixed with $90 \%$ methanol for $10 \mathrm{~min}$ and stained with $0.1 \%$ crystal violet for $10 \mathrm{~min}$, and averaged across five random fields at x100 magnification using an inverted microscope (Olympus Corporation, Tokyo, Japan).

Target prediction of miRNAs. TargetScan (http://www. targetscan.org/) was adopted to identify the potential targets of miR-452.

Western blot analysis. Following incubation at $37^{\circ} \mathrm{C}$ for $72 \mathrm{~h}$ post-transfection, cells were lysed using radioimmunoprecipitation assay lysis buffer (Beyotime Institute of Biotechnology, Haimen, China) supplemented with $0.1 \mathrm{mg} / \mathrm{ml}$ phenylmethylsulfonyl fluoride, $1 \mathrm{mM}$ sodium orthovanadate and $1 \mathrm{mg} / \mathrm{ml}$ aprotinin. Protein concentration was determined using a bicinchoninic acid assay kit (Beyotime Institute of Biotechnology). Equal amounts of protein $(20 \mathrm{mg})$ were separated by $10 \%$ SDS-PAGE and transferred to polyvinylidene fluoride membranes (EMD Millipore, Billerica, MA, USA) using a Bio-Rad semidry transfer system (Bio-Rad Laboratories, Inc., Hercules, CA, USA). The membranes were then blocked in 5\% skim milk in TBS-Tween-20 (TBST) for $2 \mathrm{~h}$ at room temperature, followed by incubation with the following primary antibodies: Mouse anti-human monoclonal BMI1 (1:1,000 dilution; cat no. sc-13519) and mouse anti-human GADPH (1:1,000 dilution; cat no. sc-166574) (both from Santa Cruz Biotechnology, Inc., Dallas, TX, USA) overnight at $4^{\circ} \mathrm{C}$. The membranes were washed three times with TBST and probed with goat anti-mouse horseradish peroxidase-conjugated secondary antibody (1:5,000 dilution; cat no. sc-2005; Santa 

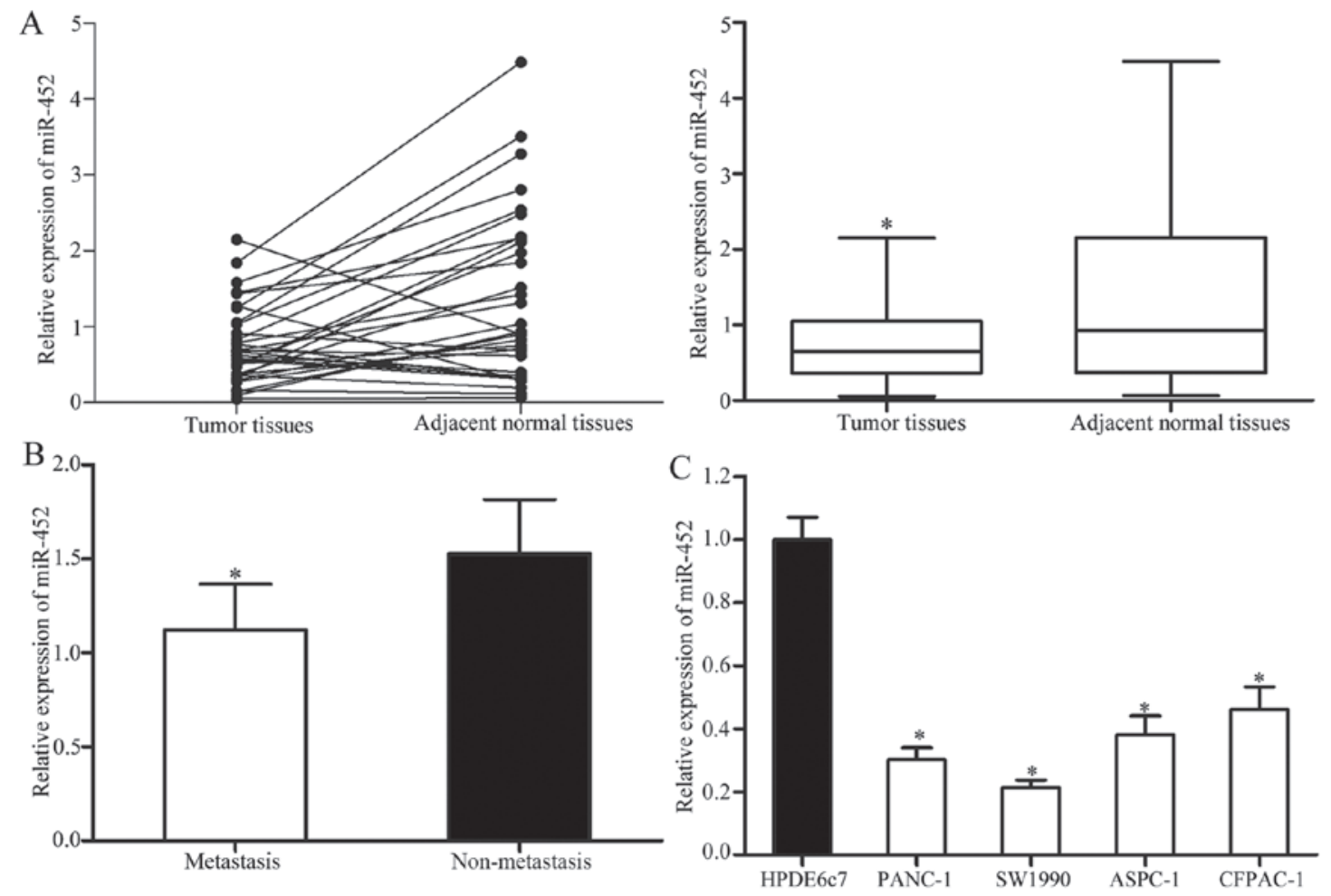

Figure 1. miR-452 expression levels in pancreatic cancer tissues and cell lines. (A) Expression levels of miR-452 in pancreatic cancer tissues and matched adjacent normal tissues. (B) Levels of miR-452 in metastatic pancreatic cancer tissues and pancreatic cancer without metastasis. (C) miR-452 expression levels in four pancreatic cancer cell lines and the human normal pancreatic cell line. ${ }^{*} \mathrm{P}<0.05$ vs. the respective controls. miR-452, microRNA-452.

Cruz Biotechnology, Inc.) at room temperature for $1 \mathrm{~h}$. Membranes were then washed three times with TBST and visualized with enhanced chemiluminescence plus reagents (Pierce; Thermo Fisher Scientific, Inc.). Protein expression was quantified using Quantity One software version 4.62 (Bio-Rad Laboratories, Inc.). GADPH was used as an internal control for BMI1 protein expression.

Dual-luciferase reporter assay. PGL3-BMI1-3'UTR wild-type (Wt) and PGL3-BMI1-3'UTR mutant (Mut) were purchased from Shanghai GenePharma Co., Ltd. Cells were seeded onto 96-well plates and transfected with miR-452 mimics or NC, and co-transfected with PGL3-BMI1-3'UTR Wt or PGL3-BMI1-3'UTR Mut using Lipofectamine 2000 according to the manufacturer's protocol. Following incubation at $37^{\circ} \mathrm{C}$ for $48 \mathrm{~h}$, cells were collected and luciferase activities were measured using a Dual-Luciferase Reporter Assay system (Promega Corporation). The relative luciferase activities were normalized to that of Renilla activity.

Statistical analysis. Data are expressed as the mean \pm standard deviation, and compared using SPSS 17.0 software (SPSS, Inc., Chicago, IL, USA). Groups were compared using two-tailed Student's t-tests or one-way analysis of variance with Student-Newman-Keuls post hoc tests. $\mathrm{P}<0.05$ was considered to indicate a statistically significant difference.

\section{Results}

miR-452 is frequently downregulated in pancreatic cancer tissues and cell lines. The expression levels of mature
miR-452 in the pancreatic cancer tissues and matched adjacent normal tissues were measured using RT-qPCR. The results revealed that miR-452 was significantly decreased in the pancreatic cancer tissues compared with that in matched adjacent normal tissues (Fig. 1A). In addition, the levels of miR-452 in metastatic pancreatic cancer tissues were significantly lower compared with that in pancreatic cancer without metastasis (Fig. 1B).

miR-452 expression levels were also explored in four pancreatic cancer cell lines (PANC-1, SW1990, ASPC-1 and CFPAC-1) and the human normal pancreatic cell line (HPDE6c7). Lower expression levels of miR-452 were observed in all four pancreatic cancer cell lines compared with that in the HPDE6c7 cell line (Fig. 1C). Among these cell lines, PANC-1 and SW1990 cells were selected for subsequent functional studies as they expressed relatively lower levels of miR-452.

Overexpression of miR-452 inhibits pancreatic cancer migration and invasion. Since miR-452 was downregulated in patients with metastatic pancreatic cancer, whether overexpression of miR-452 decreased migration and invasion capacity of pancreatic cancer was subsequently explored. PANC-1 and SW1990 cells were transfected with miR-452 mimics or NC. The ectopic expression efficiency of miR-452 was validated by RT-qPCR (Fig. 2A).

To clarify the effect of miR-452 on tumor metastasis, migration and invasion assays were performed with Transwell chambers. The results revealed that the migratory and invasive abilities of PANC-1 and SW1990 cells were suppressed by miR-452 overexpression (Fig. 2B). Collectively, these 



Figure 2. Overexpression of miR-452 inhibited pancreatic cancer migration and invasion. (A) Expression levels of miR-452 in PANC-1 and SW1990 cells. PANC-1 and SW1990 cells were transfected with miR-452 mimics or NC. (B) Cell migration and invasion capacity of PANC-1 and SW1990 cells was assessed by migration and invasion assays following transfection with miR-452 mimics or NC. ${ }^{*} \mathrm{P}<0.05$ vs. the respective controls. miR-452, microRNA-452; NC, negative control.

results indicated that miR-452 functioned as a tumor suppressor and contributed to suppression of metastasis of pancreatic cancer.

BMIl is a direct target gene of miR-452 in pancreatic cancer. To further elucidate the molecular mechanisms underlying the inhibitory functions of miR-452 in pancreatic cancer, TargetScan was applied to identify predicated targets. As presented in Fig. 3A, BMI1 contained the predicted binding sites for miR-452. Dual-luciferase reporter assays were then performed to investigate whether miR-452 directly targeted the 3'UTR of BMI1. The results revealed that co-transfection of miR-452 significantly decreased the activities of the firefly luciferase reporter with BMI1-3'UTR Wt, whereas the inhibition effect was abolished when the predicted 3'UTR binding sites of BMI1 were mutated (Fig. 3B).

Furthermore, RT-qPCR and western blot analysis were adopted to determine the regulatory effects of miR-452 in BMI1 expression. The results indicated that overexpression of miR-452 markedly suppressed BMI1 mRNA and protein levels in PANC-1 and SW1990 cells (Fig. 3C and D). Taken together, BMI1 was a direct target gene of miR-452 in pancreatic cancer.
Overexpression of miR-452 inhibits migration and invasion of pancreatic cancer cells via knockdown of BMII. BMI1 was verified as a direct target gene of miR-452 in pancreatic cancer. To explore whether the inhibition effects of miR-452 on migration and invasion of pancreatic cancer was achievable by knockdown of BMI1, BMI1 siRNA or NC siRNA were transfected into PANC-1 and SW1990 cells. The transfection efficiency was measured using western blot analysis (Fig. 4A). The effect of BMI1 siRNA in metastasis of pancreatic cancer was also measured. The migration and invasion assays indicated that BMI1 siRNA inhibited migration and invasion capacity of PANC-1 and SW1990 cells compared with that in NC siRNA groups (Fig. 4B). These results suggested that overexpression of miR-452 inhibited migration and invasion of pancreatic cancer, at least partially by knockdown of BMI1 expression.

\section{Discussion}

Pancreatic cancer is an aggressive tumor characterized by early and aggressive metastasis, as well as dissemination of pancreatic cancer (7). Successful management and therapy of patients with pancreatic cancer remains one of the major 


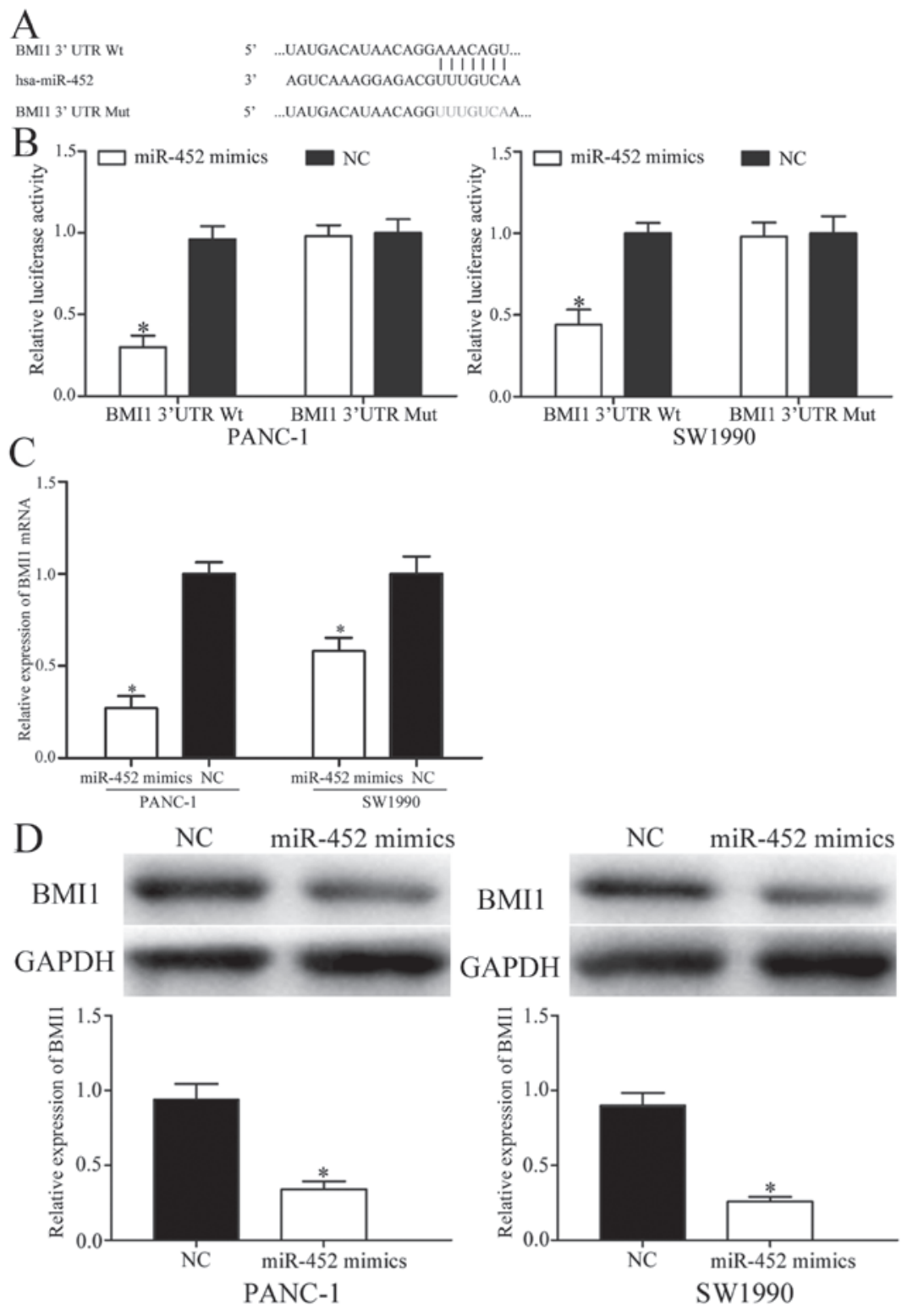

Figure 3. BMI1 was a direct target of miR-452 in pancreatic cancer. (A) The predicted binding sequences for BMI1 3'UTR with miR-452. (B) Dual-luciferase reporter assays were used to measure the interaction between miR-452 and the BMI1 3'UTR. The BMI1 3'UTR Wt or BMI1 3'UTR Mut was co-transfected with miR-452 mimics or NC and incubated for $48 \mathrm{~h}$ with PANC-1 and SW1990 cells. (C) The mRNA levels of BMI1 were detected by reverse transcription-quantitative polymerase chain reaction. PANC-1 and SW1990 cells were transfected with miR-452 mimics or NC. (D) BMI1 protein levels were measured by western blot analysis. PANC-1 and SW1990 cells were transfected with miR-452 mimics or NC. $\mathrm{P}<0.05$ vs. the respective controls. BMI1, B-cell-specific Moloney murine leukemia virus insertion site 1; miR-452, microRNA-452; 3'UTR, 3' untranslated region; NC, negative control; Wt, wild-type; Mut, mutant.

challenges in clinical oncology. It is possible to cure patients with early stage pancreatic cancer with surgical operation (20). However, the majority of patients are diagnosed at advanced stages and surgery is not possible (21). Therefore, there is an urgent requirement for novel therapeutic treatments for patients with pancreatic cancer. A number of studies have indicated that miRNAs contribute to the carcinogenesis and progression of numerous types of human cancer via targeting multiple mRNAs (22-24). Therefore, identification of specific miRNAs may provide therapeutic implications and may be exploited to improve treatments for patients with pancreatic cancer.

The present study is the first to investigate the expression, biological functions and molecular mechanism of miR-452 in pancreatic cancer. It was demonstrated that miR-452 was significantly downregulated in pancreatic cancer tissues and cell lines. In addition, the levels of miR-452 in metastatic pancreatic cancer tissues were significantly lower compared with those in pancreatic cancer without metastasis. Functional studies indicated that overexpression of miR-452 suppressed the migration and invasion of pancreatic cancer. In addition, BMI1 was identified as a direct target gene of miR-452 in pancreatic cancer. Thus, miR-452 acted as a tumor suppressor in pancreatic cancer and its downregulation enhanced metastasis of pancreatic cancer.

miR-452 has been studied in numerous types of human cancer $(15,16,25)$. For example, He et al (15) reported that the expression levels of miR-452 were decreased in non-small-cell lung cancer, and low expression levels of miR-452 were 

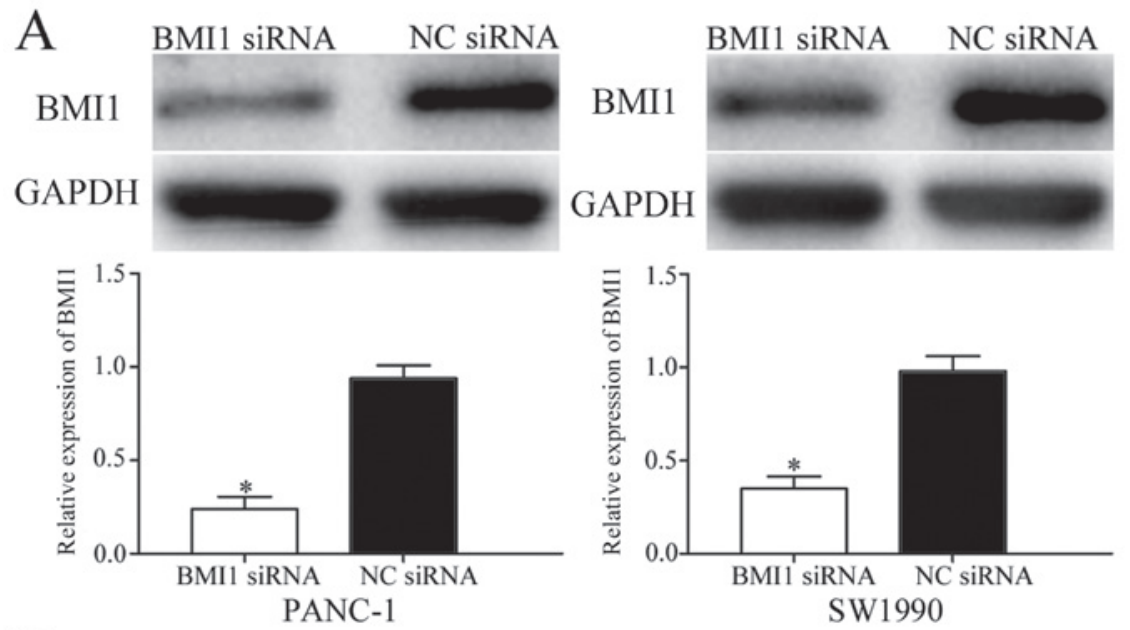

B Migration assay

Invasion assay

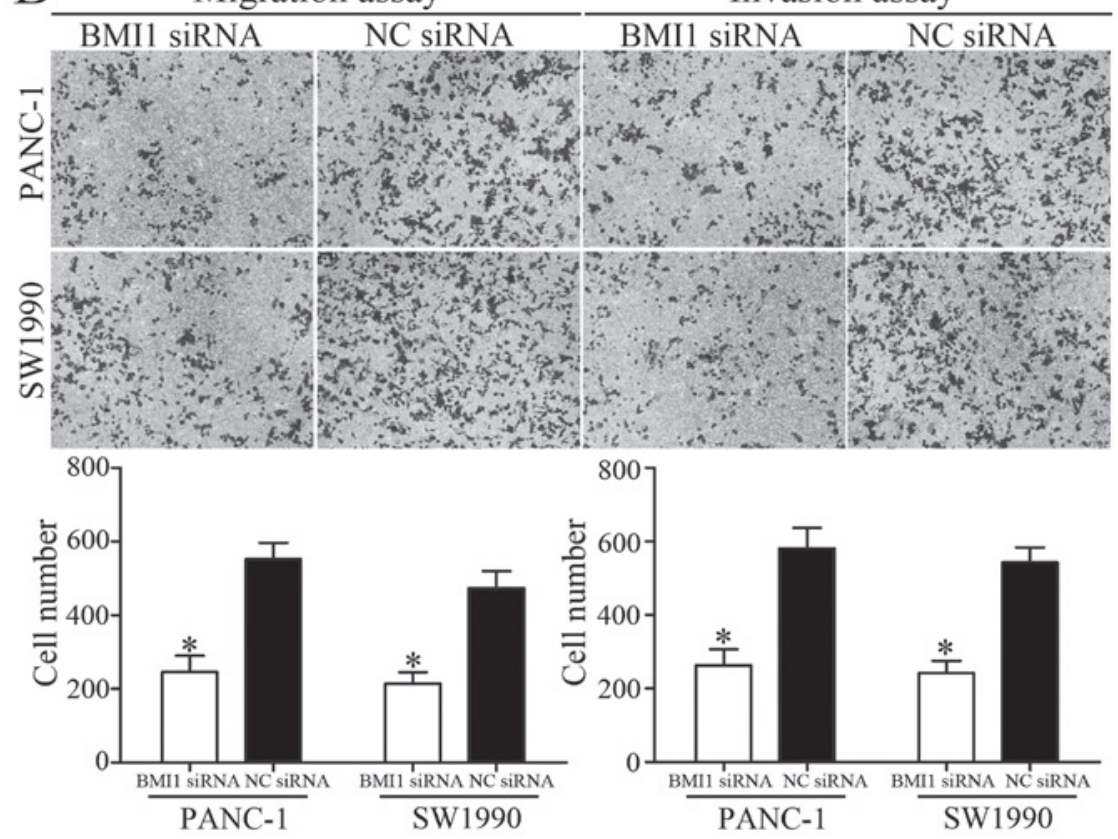

Figure 4. Knockdown of BMI1 inhibited pancreatic cancer migration and invasion. (A) BMI1 protein levels were measured by western blot analysis. PANC-1 and SW1990 cells were transfected with BMI1 siRNA or NC siRNA. (B) Cell migration and invasion of PANC-1 and SW1990 cells was assessed using Transwell migration and invasion assays following transfection with BMI1 siRNA or NC siRNA. ${ }^{*}<0.05$ vs. the respective controls. BMI1, B-cell-specific Moloney murine leukemia virus insertion site 1; siRNA, small interfering RNA; NC, negative control.

associated with advanced tumor stage and more extent of lymph nodes metastasis. Functional studies have revealed that under-expression of miR-452 may induce cell invasion of non-small-cell lung cancer (15). In addition, miR-452 was revealed to negatively regulate BMI1 expression by binding to its 3'UTR, and the enhanced cell invasion induced by downregulated miR-452 may be eliminated by knockdown of BMI1 (15). In breast cancer, miR-452 was downregulated in adriamycin-resistant MCF-7 cells compared with in parental MCF-7 cells. miR-452 mediated chemosensitivity to adriamycin in human breast cancer cells (16). Furthermore, bioinformatics analysis, RT-qPCR and western blot analysis have demonstrated that miR-452 may be at least in part be involved in adriamycin-resistance of breast cancer via the targeting of insulin-like growth factor-1 (16). Liu et al (25) revealed that miR-452 was significantly downregulated in glioma tissues and cell lines, and miR-452 levels were associated with World Health Organization grades and patient survival. Additional studies revealed that miR-452 targeted BMI1, lymphoid enhancer-binding factor 1 and $\mathrm{T}$ cell factor 4 to decrease glioma stem-like phenotypes in vitro and inhibit glioma carcinogenesis in vivo (21).

miR-452 is regarded as a tumor suppressor in non-small cell lung cancer (15), breast cancer (16) and glioma (25). However, in hepatocellular carcinoma, miR-452 was markedly upregulated in tumor tissues and cell lines (18). Ectopic expression of miR-452 enhanced cell proliferation, promoted transition from phase G1 to $\mathrm{S}$ in the cell cycle and suppressed apoptosis, migration and invasion of hepatocellular carcinoma. In addition, miR-452 decreased cyclin-dependent kinase inhibitor 1B (CDKN1B) mRNA and protein expression by directly targeting the 3'UTR of CDKN1B; the knockdown of CDKN1B resembled the phenotype resulting from overexpression of miR-452 expression (18). miR-452 was also 
demonstrated to be an oncogene in esophageal cancer (26) and urothelial carcinoma (27). These conflicting studies indicated that the expression and functions of miR-452 in human cancers have tissue specificity. It may be explained by distinct context of various microenvironments, and the imperfect complementarity of the interactions between miRNAs and target mRNAs (28). In the present study, miR-452 was demonstrated to act as a tumor suppressor in pancreatic cancer by inhibiting cell migration and invasion. The present study expanded the expression and functions of miR-452 in human cancers.

Identifying the target genes of miRNA is essential for understanding its functions in carcinogenesis and progression. In the present study, BMI1 was revealed to be the direct target gene of miR-452 in pancreatic cancer. BMI1, a member of the Polycomb group genes, was first demonstrated as an oncogene in murine lymphoma (29). Previous studies have suggested that BMI1 is highly expressed in numerous human cancers, including lung cancer (30), prostate cancer (31) and colorectal cancer (32). Additional studies have demonstrated that BMI1 serves important functions in a number of biological processes, including the cell cycle, apoptosis, senescence and proliferation (33-35). In pancreatic cancer, BMI1 is upregulated and expression levels of BMI1 are associated with proliferation, survival and poor prognosis of patients with pancreatic cancer (36). Furthermore, BMI1 functioned as an oncogene in pancreatic cancer by promoting chemoresistance, invasion and tumorigenesis of pancreatic cancer (37). The results of the present study were in accordance with the aforementioned results. The present study revealed that knockdown of BMI1 suppressed migration and invasion of pancreatic cancer cells, which is similar to the effects of miR-452 in pancreatic cancer.

In summary, the present study demonstrated that miR-452 was downregulated in pancreatic cancer tissues, particularly in metastatic tumors and pancreatic cancer cell lines. Ectopic expression of miR452 may suppress migration and invasion of pancreatic cancer cells through directly targeting BMI1. These results indicated that miR-452 may be useful as a novel potential therapeutic treatment for pancreatic cancer to block metastasis.

\section{References}

1. Siegel R, Naishadham D and Jemal A: Cancer statistics, 2013. CA Cancer J Clin 63: 11-30, 2013.

2. Siegel RL, Miller KD and Jemal A: Cancer statistics, 2015. CA Cancer J Clin 65: 5-29, 2015.

3. Moir J, White SA, French JJ, Littler P and Manas DM: Systematic review of irreversible electroporation in the treatment of advanced pancreatic cancer. Eur J Surg Oncol 40: 1598-1604, 2014.

4. Burkey MD, Feirman S, Wang H, Choudhury SR, Grover S and Johnston FM: The association between smokeless tobacco use and pancreatic adenocarcinoma: A systematic review. Cancer Epidemiol 38: 647-653, 2014.

5. Hou BH, Jian ZX, Cui P, Li SJ, Tian RQ and Ou JR: miR-216a may inhibit pancreatic tumor growth by targeting JAK2. FEBS Lett 589: 2224-2232, 2015.

6. Huang $\mathrm{C}$ and Xie K: Analysis of the potential for pancreatic cancer metastasis in vitro and in vivo. Methods Mol Biol 980: 301-319, 2013.

7. Su D, Yamaguchi K and Tanaka M: The characteristics of disseminated tumor cells in pancreatic cancer: A black box needs to be explored. Pancreatology 5: 316-324, 2005.

8. Qin Y, Dang X, Li W and Ma Q: miR-133a functions as a tumor suppressor and directly targets FSCN1 in pancreatic cancer. Oncol Res 21: 353-363, 2013.
9. Hu Y, Ou Y, Wu K, Chen Y and Sun W: miR-143 inhibits the metastasis of pancreatic cancer and an associated signaling pathway. Tumour Biol 33: 1863-1870, 2012.

10. Tréhoux S, Lahdaoui F, Delpu Y, Renaud F, Leteurtre E, Torrisani J, Jonckheer N and Van Seuningen I: Micro-RNAs miR-29a and miR-330-5p function as tumor suppressors by targeting the MUC1 mucin in pancreatic cancer cells. Biochim Biophys Acta 1853: 2392-2403, 2015.

11. Guled M and Knuutila S: MicroRNAs and cancer. Duodecim 129: 1661-1669, 2013 (In Finnish).

12. Ambros V: The functions of animal microRNAs. Nature 431: 350-355, 2004.

13. Ryan BM, Robles AI and Harris CC: Genetic variation in microRNA networks: The implications for cancer research. Nat Rev Cancer 10: 389-402, 2010.

14. Cimmino A, Calin GA, Fabbri M, Iorio MV, Ferracin M, Shimizu M, Wojcik SE, Aqeilan RI, Zupo S, Dono M, et al: miR-15 and miR-16 induce apoptosis by targeting BCL2. Proc Natl Acad Sci USA 102: 13944-13949, 2005.

15. He Z, Xia Y, Pan C, Ma T, Liu B, Wang J, Chen L and Chen Y: Up-regulation of miR-452 inhibits metastasis of non-small cell lung cancer by regulating BMI1. Cell Physiol Biochem 37: 387-398, 2015.

16. Hu Q, Gong JP, Li J, Zhong SL, Chen WX, Zhang JY, Ma TF, Ji H, Lv MM, Zhao JH and Tang JH: Down-regulation of miRNA-452 is associated with adriamycin-resistance in breast cancer cells. Asian Pac J Cancer Prev 15: 5137-5142, 2014.

17. Puerta-Gil P, Garcia-Baquero R, Jia AY, Ocaña $S$, Alvarez-Múgica M, Alvarez-Ossorio JL, Cordon-Cardo C, Cava F and Sánchez-Carbayo M: miR-143, miR-222, and miR-452 are useful as tumor stratification and noninvasive diagnostic biomarkers for bladder cancer. Am J Pathol 180: 1808-1815, 2012.

18. Zheng Q, Sheng Q, Jiang C, Shu J, Chen J, Nie Z, Lv Z and Zhang Y: MicroRNA-452 promotes tumorigenesis in hepatocellular carcinoma by targeting cyclin-dependent kinase inhibitor 1B. Mol Cell Biochem 389: 187-195, 2014.

19. Livak KJ and Schmittgen TD: Analysis of relative gene expression data using real-time quantitative PCR and the 2(Delta Delta C(T)) method. Methods 25: 402-408, 2001.

20. Link KH, Leder G, Formentini A, Fortnagel G, Kornmann M, Schatz M and Beger HG: Surgery and multimodal treatments in pancreatic cancer-a review on the basis of future multimodal treatment concepts. Gan To Kagaku Ryoho 26: 10-40, 1999.

21. Vychytilova-Faltejskova P, Kiss I, Klusova S, Hlavsa J, Prochazka V, Kala Z, Mazanec J, Hausnerova J, Kren L, Hermanova M, et al: miR-21, miR-34a, miR-198 and miR-217 as diagnostic and prognostic biomarkers for chronic pancreatitis and pancreatic ductal adenocarcinoma. Diagn Pathol 10: 38, 2015.

22. Osada $\mathrm{H}$ and Takahashi T: MicroRNAs in biological processes and carcinogenesis. Carcinogenesis 28: 2-12, 2007.

23. Yonemori K, Kurahara $\mathrm{H}$, Maemura $\mathrm{K}$ and Natsugoe $\mathrm{S}$ : MicroRNA in pancreatic cancer. J Hum Genet 62: 33-40, 2017.

24. Zhu M, Xu Z, Wang K, Wang N and Li Y: microRNA and gene networks in human pancreatic cancer. Oncol Lett 6: 1133-1139, 2013.

25. Liu L, Chen K, Wu J, Shi L, Hu B, Cheng S, Li M and Song L: Downregulation of miR-452 promotes stem-like traits and tumorigenicity of gliomas. Clin Cancer Res 19: 3429-3438, 2013.

26. Liu SG, Qin XG, Zhao BS, Qi B, Yao WJ, Wang TY, Li HC and Wu XN: Differential expression of miRNAs in esophageal cancer tissue. Oncol Lett 5: 1639-1642, 2013.

27. Veerla S, Lindgren D, Kvist A, Frigyesi A, Staaf J, Persson H, Liedberg F, Chebil G, Gudjonsson S, Borg A, et al: miRNA expression in urothelial carcinomas: Important roles of miR-10a, miR-222, miR-125b, miR-7 and miR-452 for tumor stage and metastasis and frequent homozygous losses of miR-31. Int J Cancer 124: 2236-2242, 2009.

28. Yu Z, Ni L, Chen D, Zhang Q, Su Z, Wang Y, Yu W, Wu X, Ye J, Yang S, et al: Identification of miR-7 as an oncogene in renal cell carcinoma. J Mol Histol 44: 669-677, 2013.

29. van Lohuizen M, Verbeek S, Scheijen B, Wientjens E, van der Gulden $\mathrm{H}$ and Berns A: Identification of cooperating oncogenes in $\mathrm{E}$ mu-myc transgenic mice by provirus tagging. Cell 65: 737-752, 1991.

30. Xiong D, Ye Y, Fu Y, Wang J, Kuang B, Wang H, Wang X, $\mathrm{Zu} \mathrm{L}$, Xiao G, Hao M and Wang J: Bmi-1 expression modulates non-small cell lung cancer progression. Cancer Biol Ther 16: 756-763, 2015. 
31. Wolters T, Vissers KJ, Bangma $\mathrm{CH}$, Schröder $\mathrm{FH}$ and van Leenders GJ: The value of EZH2, p27(kip1), BMI-1 and MIB-1 on biopsy specimens with low-risk prostate cancer in selecting men with significant prostate cancer at prostatectomy. BJU Int 106: 280-286, 2010.

32. Zhang X, Yang X, Zhang Y, Liu X, Zheng G, Yang Y, Wang L, Du L and Wang C: Direct serum assay for cell-free bmi-1 mRNA and its potential diagnostic and prognostic value for colorectal cancer. Clin Cancer Res 21: 1225-1233, 2015.

33. Park IK, Morrison SJ and Clarke MF: Bmi1, stem cells, and senescence regulation. J Clin Invest 113: 175-179, 2004.

34. Lee K, Adhikary G, Balasubramanian S, Gopalakrishnan R, McCormick T, Dimri GP, Eckert RL and Rorke EA: Expression of Bmi-1 in epidermis enhances cell survival by altering cell cycle regulatory protein expression and inhibiting apoptosis. J Invest Dermatol 128: 9-17, 2008.
35. Pasini D, Bracken AP and Helin K: Polycomb group proteins in cell cycle progression and cancer. Cell Cycle 3: 396-400, 2004.

36. Song W, Tao K, Li H, Jin C, Song Z, Li J, Shi H, Li X, Dang Z and Dou K: Bmi-1 is related to proliferation, survival and poor prognosis in pancreatic cancer. Cancer Sci 101: 1754-1760, 2010.

37. Yin T, Wei H, Leng Z, Yang Z, Gou S, Wu H, Zhao G, Hu X and Wang C: Bmi-1 promotes the chemoresistance, invasion and tumorigenesis of pancreatic cancer cells. Chemotherapy 57: 488-496, 2011. 